\title{
Some General Integral Operator Inequalities Associated with $\varphi$-Quasiconvex Functions
}

\author{
Young Chel Kwun, ${ }^{1}$ Moquddsa Zahra, ${ }^{2}$ Ghulam Farid (iD, ${ }^{3}$ Praveen Agarwal (D, ${ }^{4}$ \\ and Shin Min Kang ${ }^{5}$
}

${ }^{1}$ Department of Mathematics, Dong-A University, Busan 49315, Republic of Korea

${ }^{2}$ Department of Mathematics, University of Wah, Wah Cantt, Pakistan

${ }^{3}$ Department of Mathematics, COMSATS University Islamabad, Attock Campus, Pakistan

${ }^{4}$ Anand International College of Engineering, Near Kanota Agra Road, Jaipur 303012, Rajasthan, India

${ }^{5}$ Center for General Education, China Medical University, Taichung 40402, Taiwan

Correspondence should be addressed to Ghulam Farid; faridphdsms@hotmail.com and Shin Min Kang; smkang@gnu.ac.kr

Received 21 November 2020; Revised 15 December 2020; Accepted 18 December 2020; Published 8 January 2021

Academic Editor: HwaJoon Kim

Copyright (c) 2021 Young Chel Kwun et al. This is an open access article distributed under the Creative Commons Attribution License, which permits unrestricted use, distribution, and reproduction in any medium, provided the original work is properly cited.

\begin{abstract}
This paper deals with generalized integral operator inequalities which are established by using $\varphi$-quasiconvex functions. Bounds of an integral operator are established which have connections with different kinds of known fractional integral operators. All the results are deducible for quasiconvex functions. Some fractional integral inequalities are deduced.
\end{abstract}

\section{Introduction and Preliminaries}

Convex functions play a vital role in the theory of mathematical analysis. Many generalizations have been given for the convex function, for example, $\alpha$-convex, $m$-convex, $h$-convex, $\quad(\alpha, m)$-convex, $\quad(h, m)$-convex, $\quad s$-convex, $(s, m)$-convex, $\varphi$-convex, and quasiconvex functions (see $[1-10])$. We will use $\varphi$-quasiconvex functions to study the bounds of unified integral operators, and the established results are directly related with fractional integral operators in particular cases. All the fractional integral operators defined in [11-15] satisfy the results of this paper for $\varphi$-quasiconvex functions, and also the results of [16-19] are reproduced in special cases.

Definition 1 (see [20]). A function $f: J \longrightarrow \mathbb{R}$ is called convex if

$$
f\left(t x_{0}+(1-t) y_{0}\right) \leq t f\left(x_{0}\right)+(1-t) f\left(y_{0}\right), \quad t \in[0,1],
$$

holds $\forall x_{0}, y_{0} \in J$, where $J$ is an interval in $\mathbb{R}$.
Definition 2 (see [21]). A function $f: J \longrightarrow \mathbb{R}$ is called $\varphi$-quasiconvex if

$$
\begin{array}{r}
f\left(t x_{0}+(1-t) y_{0}\right) \leq \max \left\{f\left(y_{0}\right), f\left(y_{0}\right)+\varphi\left(f\left(x_{0}\right), f\left(y_{0}\right)\right)\right\}, \\
t \in[0,1],
\end{array}
$$

holds $\forall x_{0}, y_{0} \in J$, where $J$ is an interval in $\mathbb{R}$ and $\varphi: f(J) \times$ $f(J) \longrightarrow \mathbb{R}$ is a bifunction.

For $\varphi\left(x_{0}, y_{0}\right)=x_{0}-y_{0}$, (2) reduces to quasiconvex function. It is to be noted that every convex function is quasiconvex but converse does not hold.

Example 1 (see [22]). A function $f:[-2,2] \longrightarrow \mathbb{R}$ defined as

$$
f(x)= \begin{cases}1, & x \in[-2,-1] \\ x^{2}, & x \in(-1,2],\end{cases}
$$

is quasiconvex on $[-2,2]$ but not a convex function on the same interval. 
The aim of this paper is to establish integral inequalities by using $\varphi$-quasiconvex functions. The results will provide upper bounds of integral operators for $\varphi$-quasiconvex functions, which will behave like compact formulas that unify bounds of various kinds of operators already defined in literature. Next, we give some generalized fractional integral operators connected with the findings of this work.

Definition 3 (see [14]). Let $f \in L\left[x_{0}, y_{0}\right]$ and $g$ be positive and increasing function on $\left(x_{0}, y_{0}\right]$, and also, let $g$ have continuous derivative on $\left(x_{0}, y_{0}\right)$. The left and right fractional integrals of $f$ with respect to $g$ on $\left[x_{0}, y_{0}\right]$ of order $\lambda$, where $\lambda>0$, are given as follows:

$$
\begin{aligned}
& { }_{g}^{\lambda} I_{x_{0}^{+}} f(x)=\frac{1}{\Gamma(\lambda)} \int_{x_{0}}^{x}(g(x)-g(t))^{\lambda-1} g^{\prime}(t) f(t) \mathrm{d} t, \quad x>x_{0}, \\
& { }_{g}^{\lambda} I_{y_{0}^{-}} f(x)=\frac{1}{\Gamma(\lambda)} \int_{x}^{y_{0}}(g(t)-g(x))^{\lambda-1} g^{\prime}(t) f(t) \mathrm{d} t, \quad x<y_{0},
\end{aligned}
$$

where

$$
\Gamma(\lambda)=\int_{0}^{\infty} t^{\lambda-1} e^{-t} \mathrm{~d} t
$$

Definition 4 (see [23]). Let $f \in L\left[x_{0}, y_{0}\right]$ and $g$ be positive and increasing function on $\left(x_{0}, y_{0}\right]$, and also let $g$ have continuous derivative on $\left(x_{0}, y_{0}\right)$. The left and right $k$-fractional integrals of $f$ with respect to $g$ on $\left[x_{0}, y_{0}\right]$ of order $\lambda$, where $\lambda, k>0$, are given as follows:

$$
\begin{array}{ll}
{ }_{g}^{\lambda} I_{x_{0}^{+}}^{k} f(x)=\frac{1}{k \Gamma_{k}(\lambda)} \int_{x_{0}}^{x}(g(x)-g(t))^{(\lambda / k)-1} g^{\prime}(t) f(t) \mathrm{d} t, \quad x>x_{0}, \\
{ }_{g}^{\lambda} I_{y_{0}^{-}}^{k} f(x)=\frac{1}{k \Gamma_{k}(\lambda)} \int_{x}^{y_{0}}(g(t)-g(x))^{(\lambda / k)-1} g^{\prime}(t) f(t) \mathrm{d} t, \quad x<y_{0},
\end{array}
$$

where

$$
\Gamma_{k}(\lambda)=\int_{0}^{\infty} t^{\lambda-1} e^{-t^{k} / k} \mathrm{~d} t
$$

Definition 5 (see [24]). Let $f \in L_{1}\left[x_{0}, y_{0}\right]$ and $x \in\left[x_{0}, y_{0}\right]$; also, let $\Phi, \lambda, \varrho, \kappa, \gamma, \sigma \in \mathbb{C}, \quad \mathfrak{R}(\lambda), \mathfrak{R}(\varrho), \mathfrak{R}(\kappa)>0$, $\Re(\chi)>\Re(\gamma)>0$ with $p \geq 0, \vartheta>0$, and $0<k \leq \vartheta+\Re(\lambda)$, then the generalized fractional integral operators $\epsilon_{\lambda, 0, \kappa, \Phi, x_{0}^{+}}^{\gamma, 9, k, \chi} f$ and $\epsilon_{\lambda, \varrho, \kappa, \Phi, y_{0}^{-}}^{\gamma, 9, k, \chi} f$ are defined as

$$
\begin{aligned}
& \left(\epsilon_{\lambda, \varrho, \kappa, \Phi, x_{0}^{+}}^{\gamma, \vartheta, k, \chi} f\right)(x ; p)=\int_{x_{0}}^{x}(x-t)^{\varrho^{-1}} E_{\lambda, \varrho, \kappa}^{\gamma, 9, k, \chi}\left(\Phi(x-t)^{\lambda} ; p\right) f(t) \mathrm{d} t, \\
& \left(\epsilon_{\lambda, \varrho, \kappa, \Phi, y_{0}^{-}}^{\gamma, 9, k, \chi} f\right)(x ; p)=\int_{x}^{y_{0}}(t-x)^{\varrho-1} E_{\lambda, \varrho, \kappa}^{\gamma, 9, k, \chi}\left(\Phi(t-x)^{\lambda} ; p\right) f(t) \mathrm{d} t,
\end{aligned}
$$

where $E_{\lambda, \varrho, \kappa}^{\gamma, 9, k, \chi}(t ; p)$ is given by

$$
E_{\lambda, \varrho, \kappa}^{\gamma, \vartheta, k, \chi}(t ; p)=\sum_{n=0}^{\infty} \frac{\beta_{p}(\gamma+n k, \chi-\gamma)}{\beta(\gamma, \chi-\gamma)} \frac{(\chi)_{n k}}{\Gamma(\lambda n+\varrho)} \frac{t^{n}}{(\kappa)_{n \vartheta}} .
$$

In [11], Farid defined a unified integral operator and proved the boundedness, linearity, and continuity of these integrals. It is given in the following definition.

Definition 6 (see [11]). Let $f, g:\left[x_{0}, y_{0}\right] \longrightarrow \mathbb{R}$ where $0<x_{0}<y_{0}$ be the functions such that $f$ is positive and integrable over $\left[x_{0}, y_{0}\right]$ and $g$ is differentiable and strictly increasing. Also, let $\Psi / x$ be an increasing function on $\left[x_{0}, \infty\right)$ and $\varrho, \kappa, \gamma, \chi \in \mathbb{C}, p, \lambda, \vartheta \geq 0$ and $0<k \leq \vartheta+\lambda$. Then, for $x \in\left[x_{0}, y_{0}\right]$, the left and right integral operators are defined as

$$
\left({ }_{g} F_{\lambda, \varrho, \kappa, x_{0}^{+}}^{\Psi, \gamma, \vartheta, k, \chi} f\right)(x, \Phi ; p)=\int_{x_{0}}^{x} G_{x}^{y}\left(E_{\lambda, \varrho, \kappa}^{\gamma, \vartheta, k, \chi}, g ; \Psi\right) g^{\prime}(y) f(y) \mathrm{d} y,
$$

$$
\left({ }_{g} F_{\lambda, \varrho, \kappa, y_{0}^{-}}^{\Psi, \gamma, \vartheta, k, \chi} f\right)(x, \Phi ; p)=\int_{x}^{y_{0}} G_{y}^{x}\left(E_{\lambda,\rangle, \kappa}^{\gamma, \vartheta, k, \chi}, g ; \Psi\right) g^{\prime}(y) f(y) \mathrm{d} y
$$

where

$$
G_{x}^{y}\left(E_{\lambda, \varrho, \kappa}^{\gamma, \vartheta, k, \chi}, g ; \Psi\right)=\frac{\Psi(g(x)-g(y))}{g(x)-g(y)} E_{\lambda, \varrho, \kappa}^{\gamma, \vartheta, k, \chi}\left(\Phi(g(x)-g(y))^{\lambda} ; p\right) .
$$

By making particular choices for $\Psi$ and $g$ and parameters involved in (9), several fractional integrals can be obtained (see [19], Remarks 6 and 7). In [19], Zhao. et al. proved the bounds of unified integral operators for quasiconvex functions stated in Theorems 1 to 4 .

Theorem 1. Consider $f:\left[x_{0}, y_{0}\right] \longrightarrow \mathbb{R}$ be a positive quasiconvex function and $g:\left[x_{0}, y_{0}\right] \longrightarrow \mathbb{R}$ be differentiable and strictly increasing function. Also, $\Psi / x$ be an increasing function on $\left[x_{0}, y_{0}\right]$ and $\varrho, \kappa, \gamma, \chi \in \mathbb{C}, p, \lambda, \nu, \vartheta \geq 0$, $0<k \leq \vartheta+\lambda$, and $0<k \leq \vartheta+\nu$. Then, for $x \in\left[x_{0}, y_{0}\right]$, we have

$$
\begin{gathered}
\left(g_{\lambda, \varrho, \kappa, x_{0}^{+}}^{\Psi, \gamma, \vartheta, k, \chi} f\right)(x, \Phi ; p)+\left(g_{\lambda, \varrho, \kappa, y_{0}^{-}}^{\Psi, \gamma, \vartheta, k, \chi} f\right)(x, \Phi ; p) \\
\leq E_{\lambda, \varrho, \kappa}^{\gamma, \vartheta, k, \chi}\left(\Phi\left(g(x)-g\left(x_{0}\right)\right)^{\lambda} ; p\right) \Psi(g(x) \\
\quad-g\left(x_{0}\right) \max \left\{f\left(x_{0}\right), f(x)\right\} \\
\quad+E_{\gamma, \varrho, \kappa}^{\gamma, \vartheta, k, \chi}\left(\Phi\left(g\left(y_{0}\right)-g(x)\right)^{\nu} ; p\right) \Psi \\
\quad\left(g\left(y_{0}\right)-g(x)\right) \max \left\{f(x), f\left(y_{0}\right)\right\} .
\end{gathered}
$$

Theorem 2. Under the assumptions of Theorem 1, the following result holds:

$$
\begin{aligned}
& \left({ }_{g} F_{\lambda, \varrho, \kappa, x_{0}^{+}}^{\Psi, \gamma, \vartheta, k, \chi}\right)\left(y_{0}, \Phi ; p\right)+\left({ }_{g} F_{\lambda, \varrho, \kappa, y_{0}^{-}}^{\Psi, \gamma, \vartheta, k, \chi}\right)\left(x_{0}, \Phi ; p\right) \\
& \leq \Psi\left(g\left(y_{0}\right)-g\left(x_{0}\right)\right)\left(E_{\lambda, \varrho, \kappa}^{\gamma, \vartheta, k, \chi}\left(\Phi\left(g\left(y_{0}\right)-g\left(x_{0}\right)\right)^{\lambda} ; p\right)\right. \\
& \left.\quad+E_{\gamma, \varrho, \kappa}^{\gamma, \vartheta, k, \chi}\left(\Phi\left(g\left(y_{0}\right)-g\left(x_{0}\right)\right)^{\nu} ; p\right)\right) \\
& \max \left\{f\left(x_{0}\right), f\left(y_{0}\right)\right\} .
\end{aligned}
$$


Theorem 3. Along with the assumptions of Theorem 1, if $f\left(x_{0}+y_{0}-x\right)=f(x)$, then the following result holds:

$$
\begin{aligned}
& f\left(\frac{x_{0}+y_{0}}{2}\right)\left(\left({ }_{g} F_{\nu, \rho, \kappa, y_{0}^{-}}^{\Psi, \gamma, \vartheta, k, \chi} 1\right)\left(x_{0}, \Phi ; p\right)+\left({ }_{g} F_{\lambda, \varrho, \kappa, x_{0}^{+}}^{\Psi, \gamma, \vartheta, k, \chi}\right)\left(y_{0}, \Phi ; p\right)\right) \\
& \leq\left({ }_{g} F_{\nu, \varrho, k, y_{0}^{-}}^{\Psi, \gamma, \vartheta, k, \chi} f\right)\left(x_{0}, \Phi ; p\right)+\left({ }_{g} F_{\lambda, \varrho, \kappa, x_{0}^{+}}^{\Psi, \gamma, 9, k, \chi} f\right)\left(y_{0}, \Phi ; p\right) \\
& \leq \Psi\left(g\left(y_{0}\right)-g\left(x_{0}\right)\right)\left(E_{\lambda, \varrho, \kappa}^{\gamma, \vartheta, k, \chi}\left(\Phi\left(g\left(y_{0}\right)-g\left(x_{0}\right)\right)^{\lambda} ; p\right)\right. \\
& \left.+E_{\gamma, \varrho, \kappa}^{\gamma, \vartheta, k, \chi}\left(\Phi\left(g\left(y_{0}\right)-g\left(x_{0}\right)\right)^{\nu} ; p\right)\right) \\
& \max \left\{f\left(x_{0}\right), f\left(y_{0}\right)\right\} .
\end{aligned}
$$

Theorem 4. Consider $f, g:\left[x_{0}, y_{0}\right] \longrightarrow \mathbb{R}$ be two differentiable functions such that $\left|f^{\prime}\right|$ is quasiconvex and $g$ be strictly increasing for $0<x_{0}<y_{0}$. Also, $\Psi / x$ be an increasing function on $\left[x_{0}, y_{0}\right]$ and $\varrho, \kappa, \gamma, \chi \in \mathbb{C}, p, \lambda, \nu, \vartheta \geq 0$ and $0<k \leq \vartheta+\lambda$ and $0<k \leq \vartheta+\nu$. Then, for $x \in\left(x_{0}, y_{0}\right)$, we have

$$
\begin{aligned}
& \left|\left(g_{g} F_{\lambda, \varrho, \kappa, x_{0}^{+}}^{\Psi, \gamma, \vartheta, k, \chi} f * g\right)(x, \Phi ; p)+\left({ }_{g} F_{\nu, \varrho, \kappa, y_{0}^{-}}^{\Psi, \gamma, \vartheta, k, \chi} f * g\right)(x, \Phi ; p)\right| \\
& \leq E_{\lambda, \varrho, \kappa}^{\gamma, \vartheta, k, \chi}\left(\Phi\left(g(x)-g\left(x_{0}\right)\right)^{\lambda} ; p\right) \Psi\left(g(x)-g\left(x_{0}\right)\right) \\
& \quad \cdot \max \left(\left|f^{\prime}\left(x_{0}\right)\right|,\left|f^{\prime}(x)\right|\right) \\
& \quad+E_{\nu, \varrho, \kappa}^{\gamma, \vartheta, k, \chi}\left(\Phi\left(g\left(y_{0}\right)-g(x)\right)^{\nu} ; p\right) \Psi \\
& \cdot\left(g\left(y_{0}\right)-g(x)\right) \max \left(\left|f^{\prime}(x)\right|,\left|f^{\prime}\left(y_{0}\right)\right|\right),
\end{aligned}
$$

where

$$
\left({ }_{g} F_{\lambda, \varrho, \kappa, x_{0}^{+}}^{\Psi, \gamma, 9, k, \chi} f * g\right)(x, \Phi ; p)=\int_{x_{0}}^{x} G_{x}^{t}\left(E_{\lambda, \varrho, \kappa}^{\gamma, 9, k, \chi}, g ; \Psi\right) g^{\prime}(t) f^{\prime}(t) \mathrm{d} t
$$

$\left(g^{F_{\nu, 0, \kappa, y_{0}^{-}}^{\Psi, \gamma, \vartheta, k, \chi}} f * g\right)(x, \Phi ; p)=\int_{x}^{y_{0}} G_{t}^{x}\left(E_{\nu,,, \kappa}^{\gamma, 9, k, \chi}, g ; \Psi\right) g^{\prime}(t) f^{\prime}(t) \mathrm{d} t$.

All of the above results are direct consequences of the results of this paper. Also, some of the results in papers [16-18] are very special cases. In the next section, $\varphi$-quasiconvexity has been used frequently to obtain the upper bounds and the Hadamard inequality, which gives upper as well as lower bounds of unified integral operators. Also defining convolution of two functions, some bounds have been obtained for $\varphi$-quasiconvexity of $\left|f^{\prime}\right|$ of differential function $f$. In Section 3, some applications of the main results are given. In the whole paper, we use the notation

$$
\max \left\{f\left(y_{0}\right), f\left(y_{0}\right)+\varphi\left(f\left(x_{0}\right), f\left(y_{0}\right)\right)\right\}=M_{\varphi}^{f}\left(x_{0}, y_{0}\right) \text {. }
$$

\section{Main Results}

Theorem 5. Consider $f:\left[x_{0}, y_{0}\right] \longrightarrow \mathbb{R}$ is $\varphi$-quasiconvex and positive and $g:\left[x_{0}, y_{0}\right] \longrightarrow \mathbb{R}$ differentiable and strictly increasing functions. If $\Psi / x$ is increasing function on $\left[x_{0}, y_{0}\right]$ and $\quad \varrho, \kappa, \gamma, \chi \in \mathbb{C}, \quad p, \lambda, \nu, \vartheta \geq 0, \quad 0<k \leq \vartheta+\lambda \quad$ and $0<k \leq \vartheta+\nu$, then for $x \in\left[x_{0}, y_{0}\right]$, the following inequality holds:

$$
\begin{aligned}
\left(g^{F_{\lambda, \varrho, \kappa, x_{0}^{+}}^{\Psi, \gamma, \vartheta, k, \chi}} f\right)(x, \Phi ; p)+\left({ }_{g} F_{\nu, \varrho, \kappa, y_{0}^{-}}^{\Psi, \gamma, \vartheta, k, \chi} f\right)(x, \Phi ; p) \\
\leq E_{\lambda, \varrho, \kappa}^{\gamma, \vartheta, k, \chi}\left(\Phi\left(g(x)-g\left(x_{0}\right)\right)^{\lambda} ; p\right) \Psi \\
\cdot\left(g(x)-g\left(x_{0}\right)\right) M_{\varphi}^{f}\left(x_{0}, x\right) \\
+E_{\gamma, \varrho, \kappa}^{\gamma, \vartheta, k, \chi}\left(\Phi\left(g\left(y_{0}\right)-g(x)\right)^{\nu} ; p\right) \Psi \\
\cdot\left(g\left(y_{0}\right)-g(x)\right) M_{\varphi}^{f}\left(x, y_{0}\right) .
\end{aligned}
$$

Proof. For the kernel defined in (12) and the function $g$, we can write the following inequality:

$$
\begin{array}{r}
G_{x}^{t}\left(E_{\lambda, \varrho, \kappa}^{\gamma, \vartheta, k, \chi}, g ; \Psi\right) g^{\prime}(t) \leq G_{x}^{x_{0}}\left(E_{\lambda, \varrho, \kappa}^{\gamma, \vartheta, k, \chi}, g ; \Psi\right) g^{\prime}(t), \\
x \in\left(x_{0}, y_{0}\right), t \in\left[x_{0}, x\right) .
\end{array}
$$

By using $\varphi$-quasiconvexity of $f$ on $\left[x_{0}, x\right]$, one can get

$$
f(t) \leq M_{\varphi}^{f}\left(x_{0}, x\right), \quad x \in\left(x_{0}, y_{0}\right), t \in\left[x_{0}, x\right] .
$$

The following integral inequality is constituted from (21) and (22):

$$
\begin{aligned}
& \int_{x_{0}}^{x} G_{x}^{t}\left(E_{\lambda, \varrho, \kappa}^{\gamma, \vartheta, k, \chi}, g ; \Psi\right) g^{\prime}(t) f(t) \mathrm{d} t \\
& \quad \leq M_{\varphi}^{f}\left(x_{0}, x\right) G_{x}^{x_{0}}\left(E_{\lambda, \varrho, \kappa}^{\gamma, \vartheta, k, \chi}, g ; \Psi\right) \int_{x_{0}}^{x} g^{\prime}(t) \mathrm{d} t .
\end{aligned}
$$

Using (10) in the left and integrating on the right side of inequality (23), we obtain the following upper bound of the left integral operator:

$$
\begin{gathered}
\left({ }_{g} F_{\lambda, \varrho, \kappa, x_{0}^{+}}^{\Psi, \gamma, \vartheta, k, \chi} f\right)(x, \Phi ; p) \leq E_{\lambda, \varrho, \kappa}^{\gamma, \vartheta, k, \chi}\left(\Phi\left(g(x)-g\left(x_{0}\right)\right)^{\lambda} ; p\right) \Psi \\
\cdot\left(g(x)-g\left(x_{0}\right)\right) M_{\varphi}^{f}\left(x_{0}, x\right) .
\end{gathered}
$$

Now, following the similar technique for $t \in\left(x, y_{0}\right]$ and $x \in\left(x_{0}, y_{0}\right)$, we can write

$$
G_{t}^{x}\left(E_{\nu, \varrho, \kappa}^{\gamma, \vartheta, k, \chi}, g ; \Psi\right) g^{\prime}(t) \leq G_{y_{0}}^{x}\left(E_{\nu, \varrho, \kappa}^{\gamma, \vartheta, k, \chi}, g ; \Psi\right) g^{\prime}(t) .
$$

Using $\varphi$-quasiconvexity for $t \in\left(x, y_{0}\right]$ and $x \in\left(x_{0}, y_{0}\right)$, we obtain

$$
f(t) \leq M_{\varphi}^{f}\left(x, y_{0}\right)
$$


The following integral inequality is constituted from (25) and (26):

$$
\begin{aligned}
& \int_{x}^{y_{0}} G_{t}^{x}\left(E_{\nu, \varrho, \kappa}^{\gamma, \vartheta, k, \chi}, g ; \Psi\right) g^{\prime}(t) f(t) \mathrm{d} t \leq G_{y_{0}}^{x}\left(E_{\nu, \varrho, \kappa}^{\gamma, \vartheta, k, \chi}, g ; \Psi\right) \\
& \cdot M_{\varphi}^{f}\left(x, y_{0}\right) \int_{x}^{y_{0}} g^{\prime}(t) \mathrm{d} t .
\end{aligned}
$$

Using (11) in the left and integrating on the right side of the above inequality, we obtain the following upper bound of the right integral operator:

$$
\begin{aligned}
& \left({ }_{g} F_{\nu, \varrho, \kappa, y_{0}^{-}}^{\Psi, \gamma, \vartheta, k, \chi} f\right)(x ; p) \leq E_{\nu, \varrho, \kappa}^{\gamma, \vartheta, k, \chi}\left(\Phi\left(g\left(y_{0}\right)-g(x)\right)^{\nu} ; p\right) \Psi \\
& \quad \cdot\left(g\left(y_{0}\right)-g(x)\right) M_{\varphi}^{f}\left(x, y_{0}\right) .
\end{aligned}
$$

By summing (24) and (28), the inequality (20) can be obtained.

Corollary 1. Using $\lambda=v$ in (20), we get the following result:

$$
\begin{aligned}
\left(g^{F_{\lambda, \varrho, \kappa, x_{0}^{+}}^{\Psi, \gamma, \vartheta}} f\right)(x, \Phi ; p)+\left(g_{\lambda, \varrho, \kappa, y_{0}^{-}}^{\Psi, \gamma, \vartheta} f\right)(x, \Phi ; p) \\
\leq E_{\lambda, \varrho, \kappa}^{\gamma, \vartheta, k, \chi}\left(\Phi\left(g(x)-g\left(x_{0}\right)\right)^{\lambda} ; p\right) \Psi\left(g(x)-g\left(x_{0}\right)\right. \\
\cdot M_{\varphi}^{f}\left(x_{0}, x\right) \\
+E_{\lambda, \varrho, \kappa}^{\gamma, \vartheta, k, \chi}\left(\Phi\left(g\left(y_{0}\right)-g(x)\right)^{\lambda} ; p\right) \Psi \\
\cdot\left(g\left(y_{0}\right)-g(x)\right) M_{\varphi}^{f}\left(x, y_{0}\right) .
\end{aligned}
$$

\section{Remark 1}

(i) For $\varphi\left(x_{0}, y_{0}\right)=x_{0}-y_{0}$ in (20), we obtain inequality (13) of Theorem 1 .

(ii) For $\varphi\left(x_{0}, y_{0}\right)=x_{0}-y_{0}, \Psi(x)=x^{\lambda / k}$ for the lefthand integral and $\Psi(x)=x^{v / k}$ for the right-hand integral in (20) with $p=\Phi=0$, we obtain Theorem 2.1 in [17]

(iii) For $\lambda=\nu$ in the resulting inequality of (ii), we obtain Corollary 2.2 in [17].

(iv) For $\varphi\left(x_{0}, y_{0}\right)=x_{0}-y_{0}, \Psi(x)=x^{\lambda}$ for the lefthand integral and $\Psi(x)=x^{\nu}$ for the right-hand integral in (20) with $p=\Phi=0$, we obtain Corollary 2.3 in [17].

(v) Under the same assumptions as in (ii) along with $g$ as identity function, the result (20) reduces to Corollary 2.4 in [17].

(vi) Under the same assumptions as in (iv) along with $g$ as identity function, the result (20) reduces to Corollary 2.5 in [17].

(vii) Under the same assumptions as in (ii), if $f$ is increasing on $\left[x_{0}, y_{0}\right]$, the result $(20)$ reduces to Corollary 2.6 in [17]. (viii) Under the same assumptions as in (ii), if $f$ is decreasing on $\left[x_{0}, y_{0}\right]$, the result (20) reduces to Corollary 2.7 in [17].

(iix) For $\lambda=v$ in the resulting inequality of (viii), we obtain Corollary 2.2 in [18].

Theorem 6. The following result holds under the suppositions of Theorem 5:

$$
\begin{aligned}
\left({ }_{g} F_{\lambda, \varrho, \kappa, x_{0}^{+}}^{\Psi, \gamma, \vartheta, k, \chi} f\right)\left(y_{0}, \Phi ; p\right)+\left({ }_{g} F_{\nu, \varrho, \kappa, y_{0}^{-}}^{\Psi, \gamma, \vartheta, k, \chi} f\right)\left(x_{0}, \Phi ; p\right) \\
\leq \Psi\left(g\left(y_{0}\right)-g\left(x_{0}\right)\right)\left[E_{\lambda, \varrho, \kappa}^{\gamma, \vartheta, k, \chi}\left(\Phi\left(g\left(y_{0}\right)-g\left(x_{0}\right)\right)^{\lambda} ; p\right)\right. \\
\left.\quad+E_{\gamma, \varrho, \kappa}^{\gamma, \vartheta, k, \chi}\left(\Phi\left(g\left(y_{0}\right)-g\left(x_{0}\right)\right)^{\nu} ; p\right)\right] M_{\varphi}^{f}\left(x_{0}, y_{0}\right) .
\end{aligned}
$$

Proof. Using $x=y_{0}$ in (24) and $x=x_{0}$ in (28) and then adding the obtained inequalities, we get (30).

Corollary 2. Using $\lambda=\nu$ in (30), we get the following result:

$$
\begin{aligned}
\left({ }_{g} F_{\lambda, \varrho, \kappa, x_{0}^{+}}^{\Psi, \gamma, \vartheta, k, \chi} f\right) & \left(y_{0}, \Phi ; p\right)+\left({ }_{g} F_{\lambda, \varrho, \kappa, y_{0}^{-}}^{\Psi, \gamma, \vartheta, k, \chi} f\right)\left(x_{0}, \Phi ; p\right) \\
\leq & 2 \Psi\left(g\left(y_{0}\right)-g\left(x_{0}\right)\right) \\
\cdot & E_{\lambda, \varrho, \kappa}^{\gamma, \vartheta, k, \chi}\left(\Phi\left(g\left(y_{0}\right)-g\left(x_{0}\right)\right)^{\lambda} ; p\right) M_{\varphi}^{f}\left(x_{0}, y_{0}\right) .
\end{aligned}
$$

Remark 2

(i) For $\varphi\left(x_{0}, y_{0}\right)=x_{0}-y_{0}$ in (30), we obtain inequality (14) of Theorem 2

(ii) For $\varphi\left(x_{0}, y_{0}\right)=x_{0}-y_{0}, \Psi(x)=x^{\lambda / k}$ for the lefthand integral and $\Psi(x)=x^{v / k}$ for the right-hand integral in (30) with $p=\Phi=0$, we obtain Theorem 3.1 in [17].

(iii) For $\varphi\left(x_{0}, y_{0}\right)=x_{0}-y_{0}, \Psi(x)=x^{\lambda / k}$ for the lefthand integral and $\Psi(x)=x^{v / k}$ for the right-hand integral in (31) with $p=\Phi=0$, we obtain Corollary 3.2 in [17].

(iv) For $\varphi\left(x_{0}, y_{0}\right)=x_{0}-y_{0}$, replacing $\Phi$ with $\Phi^{\prime}=\Phi /\left(y_{0}-x_{0}\right)^{\lambda}, \Psi(x)=x^{\lambda}$ for the left-hand integral, $\Psi(x)=x^{\nu}$ for the right-hand integral, and $g$ as identity function in (30), we obtain Theorem 2.1 in [18].

(v) Under the same assumptions as in (iv) along with $\lambda=k=1$, the result (30) reduces to Theorem 3.3 in [16].

Before proceeding to the next result, we will prove the following lemma. This lemma is necessary to prove the upcoming result.

Lemma 1. Let $f:\left[x_{0}, y_{0}\right] \longrightarrow \mathbb{R}$ be $\varphi$-quasiconvex function. If $f(x)=f\left(x_{0}+y_{0}-x\right)$, then the following inequality holds: 


$$
f\left(\frac{x_{0}+y_{0}}{2}\right) \leq M_{\varphi}^{f}(x, x), \quad x \in\left[x_{0}, y_{0}\right]
$$

Proof. Using $\varphi$-quasiconvexity of the function $f$, the upcoming inequality holds:

$$
\begin{aligned}
& f\left(\frac{x_{0}+y_{0}}{2}\right) \leq \max \left[\begin{array}{c}
f\left(\frac{x-x_{0}}{y_{0}-x_{0}} x_{0}+\frac{y_{0}-x}{y_{0}-x_{0}} y_{0}\right), f\left(\frac{x-x_{0}}{y_{0}-x_{0}} x_{0}+\frac{y_{0}-x}{y_{0}-x_{0}} y_{0}\right) \\
+\varphi\left(f\left(\frac{x-x_{0}}{y_{0}-x_{0}} y_{0}+\frac{y_{0}-x}{y_{0}-x_{0}} x_{0}\right), f\left(\frac{x-x_{0}}{y_{0}-x_{0}} x_{0}+\frac{y_{0}-x}{y_{0}-x_{0}} y_{0}\right)\right)
\end{array}\right], \\
& f\left(\frac{x_{0}+y_{0}}{2}\right) \leq M_{\varphi}^{f}\left(x, x_{0}+y_{0}-x\right) .
\end{aligned}
$$

Using $f\left(x_{0}+y_{0}-x\right)=f(x)$ in above inequality, we get the required inequality.

Remark 3. Using $\varphi\left(x_{0}, y_{0}\right)=x_{0}-y_{0}$, (32) coincides with Lemma 1 in [19].
Theorem 7. Along with the assumptions of Theorem 5, if $f\left(x_{0}+y_{0}-x\right)=f(x)$ and $\varphi(x, y)=x+y$, then the following results hold:

$$
\begin{aligned}
& \frac{1}{3} f\left(\frac{x_{0}+y_{0}}{2}\right)\left(\left(g_{g, \varrho, \kappa, x_{0}^{+}}^{F^{\Psi, \gamma, \vartheta, k}} 1\right)\left(y_{0}, \Phi ; p\right)+\left({ }_{g} F_{\nu, \varrho, \kappa, y_{0}^{-}}^{\Psi, \gamma, \vartheta, k, \chi} 1\right)\left(x_{0}, \Phi ; p\right)\right) \\
& \leq\left({ }_{g} F_{\lambda, \varrho, \kappa, x_{0}^{+}}^{\Psi, \gamma, \vartheta, k} f\right)\left(y_{0}, \Phi ; p\right)+\left({ }_{g} F_{\nu, \varrho, \kappa, y_{0}^{-}}^{\Psi, \gamma, \vartheta, k} f\right)\left(x_{0}, \Phi ; p\right) \leq \Psi\left(g\left(y_{0}\right)-g\left(x_{0}\right)\right) \\
& \cdot\left(E_{\lambda, \varrho, \kappa}^{\gamma, \vartheta, k, \chi}\left(\Phi\left(g\left(y_{0}\right)-g\left(x_{0}\right)\right)^{\lambda} ; p\right)+E_{\nu, \varrho, \kappa}^{\gamma, \vartheta, k, \chi}\left(\Phi\left(g\left(y_{0}\right)-g\left(x_{0}\right)\right)^{\nu} ; p\right)\right) M_{\varphi}^{f}\left(x_{0}, y_{0}\right) \\
& f\left(\frac{x_{0}+y_{0}}{2}\right)\left(\left({ }_{g} F_{\lambda, \varrho, \kappa, x_{0}^{+}}^{\Psi, \gamma, \vartheta, k, \chi} 1\right)\left(y_{0}, \Phi ; p\right)+\left({ }_{g} F_{\gamma, \varrho, \kappa, y_{0}^{-}}^{\Psi,, \gamma, \vartheta, k, \chi} 1\right)\left(x_{0}, \Phi ; p\right)\right) \\
& \leq\left({ }_{g} F_{\lambda, \varrho, \kappa, x_{0}^{+}}^{\Psi, \gamma, \vartheta, k} f\right)\left(y_{0}, \Phi ; p\right)+\left({ }_{g} F_{\gamma, \varrho, \kappa, y_{0}^{-}}^{\Psi, \gamma, \vartheta, k} f\right)\left(x_{0}, \Phi ; p\right) \leq \Psi\left(g\left(y_{0}\right)-g\left(x_{0}\right)\right) \\
& \cdot\left(E_{\lambda, \varrho, \kappa}^{\gamma, \vartheta, k, \chi}\left(\Phi\left(g\left(y_{0}\right)-g\left(x_{0}\right)\right)^{\lambda} ; p\right)+E_{\gamma, \varrho, \kappa}^{\gamma, \vartheta, k, \chi}\left(\Phi\left(g\left(y_{0}\right)-g\left(x_{0}\right)\right)^{\nu} ; p\right)\right) M_{\varphi}^{f}\left(x_{0}, y_{0}\right) \text {, }
\end{aligned}
$$

provided $M_{\varphi}^{f}(x, x)=f(x)+\varphi(f(x), f(x))$ or $M_{\varphi}^{f}(x, x)=$ $f(x)$.

$$
f(x) \leq M_{\varphi}^{f}\left(x_{0}, y_{0}\right), \quad x \in\left(x_{0}, y_{0}\right) .
$$

Proof. From the kernel defined in (12) and the function $g$, we can write

$$
\begin{array}{r}
G_{x}^{x_{0}}\left(E_{\nu, \varrho, \kappa}^{\gamma, \vartheta, k, \chi}, g ; \Psi\right) g^{\prime}(x) \leq G_{y_{0}}^{x_{0}}\left(E_{\nu, \varrho, \kappa}^{\gamma, \vartheta, k, \chi}, g ; \Psi\right) g^{\prime}(x), \\
x \in\left(x_{0}, y_{0}\right) .
\end{array}
$$

Using $\varphi$-quasiconvexity of $f$ on $\left[x_{0}, y_{0}\right]$, we have (37):

The following inequality is constituted from (36) and

$$
\begin{aligned}
& \int_{x_{0}}^{y_{0}} G_{x}^{x_{0}}\left(E_{\nu, \varrho, \kappa}^{\gamma, \vartheta, k, \chi}, g ; \Psi\right) g^{\prime}(x) f(x) \mathrm{d} x \\
& \quad \leq G_{y_{0}}^{x_{0}}\left(E_{\nu, \varrho, \kappa}^{\gamma, \vartheta, k, \chi}, g ; \Psi\right) M_{\varphi}^{f}\left(x_{0}, y_{0}\right) \int_{x_{0}}^{y_{0}} g^{\prime}(x) \mathrm{d} x .
\end{aligned}
$$

Using (11) in the left and integrating on the right side of the above inequality, we obtain the following upper bound of the right integral operator: 


$$
\begin{aligned}
& \left({ }_{g} F_{\nu, \rho, \kappa, y_{0}^{-}}^{\Psi, \gamma, \vartheta, k} f\right)\left(x_{0}, \Phi ; p\right) \leq E_{\nu, \varrho, \kappa}^{\gamma, \vartheta, k, \chi}\left(\Phi\left(g\left(y_{0}\right)-g\left(x_{0}\right)\right)^{\nu} ; p\right) \Psi \\
& \quad \cdot\left(g\left(y_{0}\right)-g\left(x_{0}\right)\right) M_{\varphi}^{f}\left(x_{0}, y_{0}\right) .
\end{aligned}
$$

Also,

$$
\begin{array}{r}
G_{y_{0}}^{x}\left(E_{\lambda, \mathrm{e}, \kappa}^{\gamma, 9, k, \chi}, g ; \Psi\right) g^{\prime}(x) \leq G_{y_{0}}^{x_{0}}\left(E_{\lambda, \mathrm{e}, \kappa}^{\gamma, 9, k, \chi}, g ; \Psi\right) g^{\prime}(x), \\
x \in\left(x_{0}, y_{0}\right) .
\end{array}
$$

From (37) and (40), we get

$$
\begin{aligned}
& \int_{x_{0}}^{y_{0}} G_{y_{0}}^{x}\left(E_{\lambda, \mathrm{e}, \kappa}^{\gamma, 9, k, \chi}, g ; \Psi\right) g^{\prime}(x) f(x) \mathrm{d} x \\
& \quad \leq G_{y_{0}}^{x_{0}}\left(E_{\lambda, \varrho, \kappa}^{\gamma, \vartheta, k, \chi}, g ; \Psi\right) M_{\varphi}^{f}\left(x_{0}, y_{0}\right) \int_{x_{0}}^{y_{0}} g^{\prime}(x) \mathrm{d} x .
\end{aligned}
$$

Using (10) in the left and integrating on the right side of the above inequality, we obtain the following upper bound of the left integral operator:

$$
\begin{aligned}
& \left({ }_{g} F_{\lambda, \mathrm{e}, \kappa, x_{0}^{+}}^{\Psi, \gamma, \vartheta} f\right)\left(y_{0}, \Phi ; p\right) \leq E_{\lambda, \varrho, \kappa}^{\gamma, \vartheta, k, \chi}\left(\Phi\left(g\left(y_{0}\right)-g\left(x_{0}\right)\right)^{\lambda} ; p\right) \Psi \\
& \quad \cdot\left(g\left(y_{0}\right)-g\left(x_{0}\right)\right) M_{\varphi}^{f}\left(x_{0}, y_{0}\right) .
\end{aligned}
$$

Now using (32) of Lemma 1, we can have

$$
\begin{aligned}
& f\left(\frac{x_{0}+y_{0}}{2}\right) G_{x}^{x_{0}}\left(E_{\gamma, \mathrm{e}, \kappa}^{\gamma, 9, k, \chi}, g ; \Psi\right) g^{\prime}(x) \\
& \quad \leq G_{x}^{x_{0}}\left(E_{\gamma, \varrho, \kappa}^{\gamma, 9, k, \chi}, g ; \Psi\right) g^{\prime}(x) M_{\varphi}^{f}(x, x) .
\end{aligned}
$$

Case 1. If $M_{\varphi}^{f}(x, x)=f(x)+\varphi(f(x), f(x))$, then by using (11), $\varphi(x, y)=x+y$ in (43), and integrating over $\left[x_{0}, y_{0}\right]$, we get

$$
\begin{aligned}
& f\left(\frac{x_{0}+y_{0}}{2}\right)\left({ }_{g} F_{\nu, \varrho, \kappa, y_{0}^{-}}^{\Psi, \gamma, \vartheta, k, \chi} 1\right)\left(x_{0}, \Phi ; p\right) \\
& \quad \leq 3\left({ }_{g} F_{\nu, \varrho, \kappa, y_{0}^{-}}^{\Psi, \gamma, \vartheta, k, \chi} f\right)\left(x_{0}, \Phi ; p\right) .
\end{aligned}
$$

In this case, we also have

$$
\begin{aligned}
& f\left(\frac{x_{0}+y_{0}}{2}\right)\left({ }_{g} F_{\lambda, \varrho, e, k, x_{0}^{+}}^{\Psi, \gamma, \vartheta, k, \chi}\right)\left(y_{0}, \Phi ; p\right) \\
& \quad \leq 3\left({ }_{g} F_{\lambda, \varrho, \kappa, \kappa, x_{0}^{+}}^{\Psi, \gamma, \vartheta, k, \chi} f\right)\left(y_{0}, \Phi ; p\right) .
\end{aligned}
$$

Case 2. If $M_{\varphi}^{f}(x, x)=f(x)$, then by using (11) in (43), we get

$$
f\left(\frac{x_{0}+y_{0}}{2}\right)\left({ }_{g} F_{v, 0, k, y_{0}^{-}}^{\Psi, \gamma, 9, k} 1\right)\left(x_{0}, \Phi ; p\right) \leq\left({ }_{g} F_{v, \varrho, \kappa, y_{0}^{\prime}}^{\Psi, \gamma, 9, k, \chi} f\right)\left(x_{0}, \Phi ; p\right) .
$$

In this case, we also have

$$
f\left(\frac{x_{0}+y_{0}}{2}\right)\left({ }_{g} F_{\lambda, \varrho, \kappa, x_{0}^{+}}^{\Psi, \gamma, 9, k, \chi}\right)\left(y_{0}, \Phi ; p\right) \leq\left({ }_{g} F_{\lambda, \mathrm{e}, \kappa, x_{0}^{+}}^{\Psi, \gamma, 9} f\right)\left(y_{0}, \Phi ; p\right) \text {. }
$$

The inequality (34) will be obtained by summing (39) with (42) and (44) with (45) and then combining the resulting inequalities. The inequality (35) will be obtained by summing (39) with (42) and (46) with (47) and then combining the resulting inequalities.

Corollary 3. For $\lambda=v$ in (34) and (35), we get the following results:

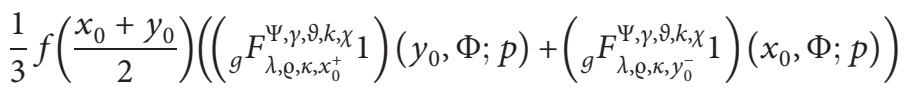

$$
\begin{aligned}
& \leq\left({ }_{g} F_{\lambda, \rho, \rho, \kappa, x_{0}^{+}}^{\Psi, \gamma, 9, k, \chi} f\right)\left(y_{0}, \Phi ; p\right)+\left({ }_{g} F_{\lambda, \mathrm{e}, \kappa, y_{0}^{-}}^{\Psi, \gamma, 9, k, \chi} f\right)\left(x_{0}, \Phi ; p\right) \\
& \leq 2 \Psi\left(g\left(y_{0}\right)-g\left(x_{0}\right)\right) E_{\lambda, \mathrm{e}, \mathrm{\gamma},}^{\gamma, 9, \chi}\left(\Phi\left(g\left(y_{0}\right)-g\left(x_{0}\right)\right)^{\lambda} ; p\right) M_{\varphi}^{f}\left(x_{0}, y_{0}\right), \\
& f\left(\frac{x_{0}+y_{0}}{2}\right)\left(\left({ }_{g} F_{\lambda, \mathrm{e}, \kappa, x_{0}^{+}}^{\Psi, \gamma, 9, k}\right)\left(y_{0}, \Phi ; p\right)+\left({ }_{g} F_{\lambda,, \mathrm{e}, \kappa, y_{0}^{-}}^{\Psi, \gamma, 9, k, \gamma}\right)\left(x_{0}, \Phi ; p\right)\right)
\end{aligned}
$$

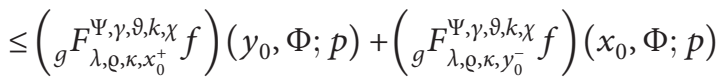

$$
\begin{aligned}
& \leq 2 \Psi\left(g\left(y_{0}\right)-g\left(x_{0}\right)\right) E_{\lambda, \mathrm{e}, \kappa}^{\gamma, 9, k}\left(\Phi\left(g\left(y_{0}\right)-g\left(x_{0}\right)\right)^{\lambda} ; p\right) M_{\varphi}^{f}\left(x_{0}, y_{0}\right) \text {. }
\end{aligned}
$$


Remark 4.

(i) For $\varphi\left(x_{0}, y_{0}\right)=x_{0}-y_{0}$ in (34), we get inequality (15) of Theorem 3.

(ii) For $\varphi\left(x_{0}, y_{0}\right)=x_{0}-y_{0}, \Psi(x)=x^{\lambda / k}$ for the lefthand integral and $\Psi(x)=x^{v / k}$ for the right-hand integral in (34) with $p=\Phi=0$, we obtain Theorem 2.16 in [17].

(iii) For $\varphi\left(x_{0}, y_{0}\right)=x_{0}-y_{0}, \Psi(x)=x^{\lambda / k}$ for the lefthand integral and $\Psi(x)=x^{v / k}$ for the right-hand integral in (48) with $p=\Phi=0$, we obtain Corollary 2.17 in [17].

(iv) For $\varphi\left(x_{0}, y_{0}\right)=x_{0}-y_{0}, \Psi(x)=x^{\lambda}$ for the lefthand integral and $\Psi(x)=x^{\nu}$ for the right-hand integral in (34) with $p=\Phi=0$, we obtain Corollary 2.18 in [17].

(v) Under the same assumptions as in (ii) along with $g$ as identity function, the result (34) reduces to Corollary 2.19 in [17].

(vi) Under the same assumptions as in (iv) along with $g$ as identity function, the result (34) reduces to Corollary 2.20 in [17].

(vii) Under the same assumptions as in (ii), if $f$ is increasing on $\left[x_{0}, y_{0}\right]$, the result (34) reduces to Corollary 2.21 in [17].

(viii) Under the same assumptions as in (ii), if $f$ is decreasing on $\left[x_{0}, y_{0}\right]$, the result (34) reduces to Corollary 2.22 in [17].

Theorem 8. Consider $f, g:\left[x_{0}, y_{0}\right] \longrightarrow \mathbb{R}$ are two differentiable functions such that $\left|f^{\prime}\right|$ is $\varphi$-quasiconvex and $g$ is strictly increasing for $0<x_{0}<y_{0}$. If $\Psi / x$ is increasing function on $\left[x_{0}, y_{0}\right]$ and $\varrho, \kappa, \gamma, \chi \in \mathbb{C}, p, \lambda, v, \vartheta \geq 0,0<k \leq \vartheta+\lambda$ and $0<k \leq \vartheta+\nu$, then for $x \in\left(x_{0}, y_{0}\right)$, the following inequality holds:

$$
\begin{gathered}
\left|\left(g_{g, \varrho, \kappa, x_{0}^{+}}^{\Psi, \gamma, \vartheta, k, \chi} f * g\right)(x, \Phi ; p)+\left(g_{g, \varrho, \kappa, y_{0}^{-}}^{\Psi, \gamma, \vartheta, k, \chi} f * g\right)(x, \Phi ; p)\right| \\
\leq E_{\lambda, \varrho, \kappa}^{\gamma, \vartheta, k, \chi}\left(\Phi\left(g(x)-g\left(x_{0}\right)\right)^{\lambda} ; p\right) \Psi\left(g(x)-g\left(x_{0}\right)\right) \\
\quad \cdot M_{\varphi}^{\left|f^{\prime}\right|}\left(x_{0}, x\right) \\
\quad+E_{\nu, \varrho, \kappa}^{\gamma, \vartheta, k, \chi}\left(\Phi\left(g\left(y_{0}\right)-g(x)\right)^{\nu} ; p\right) \Psi \\
\cdot\left(g\left(y_{0}\right)-g(x)\right) M_{\varphi}^{\left|f^{\prime}\right|}\left(x, y_{0}\right),
\end{gathered}
$$

where $\quad\left({ }_{g} F_{\lambda, 0, \kappa, x_{0}^{+}}^{\Psi, \gamma, \vartheta, k} f * g\right)(x, \Phi ; p) \quad$ and $\quad\left({ }_{g} F_{v, 0, \kappa, y_{0}^{-}}^{\Psi, \gamma, \vartheta, k, \chi} f * g\right)$ $(x, \Phi ; p)$ are defined in (17) and (18).

Proof. The $\varphi$-quasiconvexity of $\left|f^{\prime}\right|$ implies the following inequality:

$$
\left|f^{\prime}(t)\right| \leq M_{\varphi}^{\left|f^{\prime}\right|}\left(x_{0}, x\right), \quad t \in\left[x_{0}, x\right], x \in\left(x_{0}, y_{0}\right),
$$

which is equivalent to

$$
-\left(M_{\varphi}^{\left|f^{\prime}\right|}\left(x_{0}, x\right)\right) \leq f^{\prime}(t) \leq M_{\varphi}^{\left|f^{\prime}\right|}\left(x_{0}, x\right) .
$$

First consider

$$
f^{\prime}(t) \leq M_{\varphi}^{\left|f^{\prime}\right|}\left(x_{0}, x\right) .
$$

The following inequality is constituted from (21) and (53):

$$
\begin{aligned}
& \int_{x_{0}}^{x} G_{x}^{t}\left(E_{\lambda, \varrho, \kappa}^{\gamma, \vartheta, k, \chi}, g ; \Psi\right) g^{\prime}(t) f^{\prime}(t) \mathrm{d} t \\
& \quad \leq M_{\varphi}^{\left|f^{\prime}\right|}\left(x_{0}, x\right) G_{x}^{x_{0}}\left(E_{\lambda, \varrho, \kappa}^{\gamma, \vartheta, k, \chi}, g ; \Psi\right) \int_{x_{0}}^{x} g^{\prime}(t) \mathrm{d} t
\end{aligned}
$$

from which we get

$$
\begin{gathered}
\left({ }_{g} F_{\lambda, \varrho, \kappa, x_{0}^{+}}^{\Psi, \gamma, \vartheta, k, \chi} f * g\right)(x, \Phi ; p) \leq E_{\lambda, \varrho, \kappa}^{\gamma, \vartheta, k, \chi}\left(\Phi\left(g(x)-g\left(x_{0}\right)\right)^{\lambda} ; p\right) \Psi \\
\cdot\left(g(x)-g\left(x_{0}\right)\right) M_{\varphi}^{\left|f^{\prime}\right|}\left(x_{0}, x\right) .
\end{gathered}
$$

Now, we consider

$$
-\left(M_{\varphi}^{\left|f^{\prime}\right|}\left(x_{0}, x\right)\right) \leq f^{\prime}(t)
$$

Using (21) and (56), we get

$$
\begin{aligned}
& \left({ }_{g} F_{\lambda, \varrho, \kappa, x_{0}^{+}}^{\Psi, \gamma, \vartheta, k, \chi} f * g\right)(x, \Phi ; p) \geq-E_{\lambda, \varrho, \kappa}^{\gamma, \vartheta, k, \chi}\left(\Phi\left(g(x)-g\left(x_{0}\right)\right)^{\lambda} ; p\right) \Psi \\
& \cdot\left(g(x)-g\left(x_{0}\right)\right) M_{\varphi}^{\left|f^{\prime}\right|}\left(x_{0}, x\right) .
\end{aligned}
$$

Now, again using $\varphi$-quasiconvexity of $\left|f^{\prime}\right|$, we have

$$
\left|f^{\prime}(t)\right| \leq M_{\varphi}^{\left|f^{\prime}\right|}\left(x, y_{0}\right), \quad t \in\left(x, y_{0}\right], x \in\left(x_{0}, y_{0}\right) \text {. }
$$

Similarly using (25) and (58), one can obtain

$$
\begin{aligned}
& \left({ }_{g} F_{v, \varrho, \kappa, y}^{\Psi, \gamma, \vartheta, k, \chi} f * g\right)(x, \Phi ; p) \leq E_{\nu, \varrho, \kappa}^{\gamma, \vartheta, k, \chi}\left(\Phi\left(g\left(y_{0}\right)-g(x)\right)^{\nu} ; p\right) \Psi \\
& \cdot\left(g\left(y_{0}\right)-g(x)\right) M_{\varphi}^{\left|f^{\prime}\right|}\left(x, y_{0}\right),
\end{aligned}
$$

$$
\begin{aligned}
& \left.\quad{ }_{g} F_{v, \varrho, \kappa, y_{0}^{-}}^{\Psi, \gamma, \vartheta, k, \chi} f * g\right)(x, \Phi ; p) \geq-E_{\nu, \varrho, \kappa}^{\gamma, \vartheta, k, \chi} \\
& \quad \cdot\left(\Phi\left(g\left(y_{0}\right)-g(x)\right)^{\nu} ; p\right) \Psi\left(g\left(y_{0}\right)-g(x)\right) M_{\varphi}^{\left|f^{\prime}\right|}\left(x, y_{0}\right) .
\end{aligned}
$$

The inequality (50) will be obtained by summing (55), (57), (59), and (60). 
Corollary 4. For $\lambda=v$ in (50), we get the following result:

$$
\begin{aligned}
& \left|\left({ }_{g} F_{\lambda, \varrho, \kappa, x_{0}^{+}}^{\Psi, \gamma, \vartheta, k, \chi} f * g\right)(x, \Phi ; p)+\left({ }_{g} F_{\lambda, \varrho, \kappa, y_{0}^{-}}^{\Psi, \gamma, \vartheta, k, \chi} f * g\right)(x, \Phi ; p)\right| \\
& \leq E_{\lambda, \varrho, \kappa}^{\gamma, \vartheta, k, \chi}\left(\Phi\left(g(x)-g\left(x_{0}\right)\right)^{\lambda} ; p\right) \Psi\left(g(x)-g\left(x_{0}\right)\right) M_{\varphi}^{\left|f^{\prime}\right|} \\
& \quad \cdot\left(x_{0}, x\right) \\
& \quad+E_{\lambda, \varrho, \kappa}^{\gamma, \vartheta, k, \chi}\left(\Phi\left(g\left(y_{0}\right)-g(x)\right)^{\lambda} ; p\right) \Psi \\
& \quad \cdot\left(g\left(y_{0}\right)-g(x)\right) M_{\varphi}^{\left|f^{\prime}\right|}\left(x, y_{0}\right) .
\end{aligned}
$$

\section{Remark 5.}

(i) For $\varphi\left(x_{0}, y_{0}\right)=x_{0}-y_{0}$ in (50), we obtain inequality (16) of Theorem 4.

(ii) For $\varphi\left(x_{0}, y_{0}\right)=x_{0}-y_{0}, \Psi(x)=x^{\lambda / k}$ for the lefthand integral and $\Psi(x)=x^{v / k}$ for right-hand integral in (50) with $p=\Phi=0$, we obtain Theorem 2.8 in [17].

(iii) For $\varphi\left(x_{0}, y_{0}\right)=x_{0}-y_{0}, \Psi(x)=x^{\lambda / k}$ for the lefthand integral and $\Psi(x)=x^{\nu / k}$ for the right-hand integral in (61) with $p=\Phi=0$, we obtain Corollary 2.9 in [17].

(iv) For $\varphi\left(x_{0}, y_{0}\right)=x_{0}-y_{0}, \Psi(x)=x^{\lambda}$ for the lefthand integral and $\Psi(x)=x^{\nu}$ for the right-hand integral in (50) with $p=\Phi=0$, we obtain Corollary 2.10 in [17].

(v) Under the same assumptions as in (ii) along with $g$ as identity function, the result (50) reduces to Corollary 2.11 in [17].

(vi) Under the same assumptions as in (iv) along with $g$ as identity function, the result (50) reduces to Corollary 2.12 in [17].

(vii) Under the same assumptions as in (ii), if $f$ is increasing on $\left[x_{0}, y_{0}\right]$, the result (50) reduces to Corollary 2.13 in [17].

(viii) Under the same assumptions as in (ii), if $f$ is decreasing on $\left[x_{0}, y_{0}\right]$, the result (50) reduces to Corollary 2.14 in [17].

(ix) Under the same assumptions as in (ii), if in addition we put $x=x_{0}$ and $x=y_{0}$ in the left- and right-hand integrals, respectively, we obtain Theorem 3.2 in [17].

(x) For $\lambda=v$ in the resulting inequality of (ix), we obtain Corollary 3.5 in [17].

(xi) For $\lambda=k=1$ in the resulting inequality of (x), we obtain Corollary 3.6 in [17].

\section{Applications}

In this section, we present some results by applying theorems of previous section.
Proposition 1. The following result holds under the suppositions of Theorem 5:

$$
\begin{aligned}
& \Gamma(\varrho)\left(\left(\varrho_{g} I_{x_{0}^{+}} f\right)(x)+\left(\varrho_{g} I_{y_{0}^{-}} f\right)(x)\right) \leq\left(g(x)-g\left(x_{0}\right)\right)^{\varrho} \\
& \cdot M_{\varphi}^{f}\left(x_{0}, x\right)+\left(g\left(y_{0}\right)-g(x)\right)^{\varrho} M_{\varphi}^{f}\left(x, y_{0}\right) \text {. }
\end{aligned}
$$

Proof. For $\Psi(t)=t^{\varrho}, \varrho>0$ and $p=\Phi=0, \lambda=v$ with $\Psi / t$ is increasing for $\varrho \geq 1$ in the proof of Theorem 5 we get (62).

Proposition 2. The following result holds under the suppositions of Theorem 5:

$$
\begin{aligned}
& \left({ }_{x_{0}^{+}} I_{\Psi} f\right)(x)+\left({ }_{y_{0}^{-}} I_{\Psi} f\right)(x) \leq \Psi\left(x-x_{0}\right) M_{\varphi}^{f}\left(x_{0}, x\right) \\
& \quad+\Psi\left(y_{0}-x\right) M_{\varphi}^{f}\left(x, y_{0}\right) .
\end{aligned}
$$

Proof. Using $g$ as identity function, $\Phi=p=0$, and $\lambda=\nu$ in the proof of Theorem 5, we get inequality (63).

Corollary 5. For $\Psi(t)=\Gamma(\varrho) t^{\mathrm{\rho} / k} / k \Gamma_{k}(\varrho)$ in Theorem 5 , the following bound for $\varrho \geq k$ is satisfied:

$$
\begin{gathered}
\left(\varrho_{g}^{\varrho} I_{x_{0}^{+}}^{k} f\right)(x)+\left(\varrho_{g}^{\varrho} I_{y_{0}^{-}}^{k} f\right)(x) \leq \frac{1}{k \Gamma_{k}(\varrho)}\left[\left(g(x)-g\left(x_{0}\right)\right)^{\varrho / k}\right. \\
\left.\cdot M_{\varphi}^{f}\left(x_{0}, x\right)+\left(g\left(y_{0}\right)-g(x)\right)^{\varrho / k} M_{\varphi}^{f}\left(x, y_{0}\right)\right] .
\end{gathered}
$$

Corollary 6. Using $\Psi(t)=t^{\varrho}$ in (63), fractional integrals ${ }^{\varrho} I_{x_{0}^{+}} f(x)$ and ${ }^{\varrho} I_{y_{0}^{-}} f(x)$ defined in [14] are obtained which satisfy the following bound:

$$
\begin{aligned}
& \Gamma(\varrho)\left(\left({ }^{\varrho} I_{x_{0}^{+}} f\right)(x)+\left({ }^{\varrho} I_{y_{0}^{-}} f\right)(x)\right) \leq\left(x-x_{0}\right)^{\varrho} M_{\varphi}^{f}\left(x_{0}, x\right) \\
& \quad+\left(y_{0}-x\right)^{\varrho} M_{\varphi}^{f}\left(x, y_{0}\right) .
\end{aligned}
$$

Corollary 7. Using $\Psi(t)=\Gamma(\varrho) t^{\mathrm{\varrho} / k} / k \Gamma_{k}(\varrho)$ in (63), fractional integral operators ${ }^{\varrho} I_{x_{0}^{+}}^{k} f(x)$ and ${ }^{\varrho} I_{y_{0}^{-}}^{k} f(x)$ given in [26] are obtained which satisfy the following bound:

$$
\begin{aligned}
& \left({ }^{\varrho} I_{x_{0}^{+}}^{k} f\right)(x)+\left({ }^{\varrho} I_{y_{0}^{-}}^{k} f\right)(x) \leq \frac{1}{k \Gamma_{k}(\varrho)}\left[\left(x-x_{0}\right)^{\varrho / k} M_{\varphi}^{f}\left(x_{0}, x\right)\right. \\
& \left.\quad+\left(y_{0}-x\right)^{\varrho / k} M_{\varphi}^{f}\left(x, y_{0}\right)\right] .
\end{aligned}
$$

Corollary 8. Using $\Psi(t)=t^{\varrho}, \varrho>0$, and $g(x)=x^{\varepsilon} / \varepsilon, \varepsilon>0$, in (10) and (11), respectively, with $p=\Phi=0$, then fractional 
integral operators $\left({ }^{\varepsilon} I_{x_{0}^{+}}^{\mathrm{e}} f\right)(x)$ and $\left({ }^{\varepsilon} I_{y_{0}^{-}}^{\mathrm{e}} f\right)(x)$ given in [27] are obtained which satisfy the following bound:

$$
\begin{aligned}
& \left({ }^{\varepsilon} I_{x_{0}^{\dagger}}^{\varrho} f\right)(x)+\left({ }^{\varepsilon} I_{y_{0}^{-}}^{\varrho} f\right)(x) \leq \frac{1}{\varepsilon^{\varrho} \Gamma(\varrho)}\left[\left(x^{\varepsilon}-x_{0}^{\varepsilon}\right)^{\varrho} M_{\varphi}^{f}\left(x_{0}, x\right)\right. \\
& \left.\quad+\left(y_{0}^{\varepsilon}-x^{\varepsilon}\right)^{\varrho} M_{\varphi}^{f}\left(x, y_{0}\right)\right] .
\end{aligned}
$$

Corollary 9. Using $\Psi(t)=t^{\varrho}, \varrho>0$, and $g(x)=x^{s+1} / s+1$, $s>0$, in (10) and (11), respectively, with $p=\Phi=0$, then fractional integral operators $\left({ }^{s} I_{x_{0}^{+}}^{\mathrm{e}} f\right)(x)$ and $\left({ }^{s} I_{y_{0}^{-}}^{\mathrm{e}} f\right)(x)$ are obtained which satisfy the following bound:

$$
\begin{gathered}
\left({ }^{s} I_{x_{0}^{+}}^{\varrho} f\right)(x)+\left({ }^{s} I_{y_{0}^{-}}^{\varrho} f\right)(x) \leq \frac{1}{(s+1)^{\varrho} \Gamma(\varrho)}\left[\left(x^{s+1}-x_{0}^{s+1}\right)^{\varrho}\right. \\
\left.\cdot M_{\varphi}^{f}\left(x_{0}, x\right)+\left(y_{0}^{s+1}-x^{s+1}\right)^{\varrho} M_{\varphi}^{f}\left(x, y_{0}\right)\right] .
\end{gathered}
$$

$$
\begin{aligned}
& \left({ }_{k}^{s} I_{x_{0}^{+}}^{\mathrm{\rho}} f\right)(x)+\left({ }_{k}^{s} I_{y_{0}^{-}} f\right)(x) \leq \frac{1}{(s+1)^{\mathrm{\rho} / k} k \Gamma_{k}(\varrho)} \\
& \cdot\left[\left(x^{s+1}-x_{0}^{s+1}\right)^{\mathrm{\varrho} / k} M_{\varphi}^{f}\left(x_{0}, x\right)+\left(y_{0}^{s+1}-x^{s+1}\right)^{\mathrm{\rho} / k} M_{\varphi}^{f}\left(x, y_{0}\right)\right] .
\end{aligned}
$$

Corollary 11. Using $\Psi(t)=t^{\varrho}, \varrho>0, \quad g(x)=x^{\beta+s} / \beta+$ $s, \beta, s>0$, in (10) and (11), respectively, with $p=\Phi=0$, fractional integral operators $\left({ }_{\beta}^{s} I_{x^{+}}^{e^{0}} f\right)(x)$ and $\left({ }_{\beta}^{s} I_{y^{-}} f\right)(x)$ are obtained given in [13] which sattsfy the following bound:

$$
\begin{aligned}
& \left({ }_{\beta}^{s} I_{x_{0}^{+}}^{\varrho} f\right)(x)+\left({ }_{\beta}^{s} I_{y_{0}^{-}}^{\varrho} f\right)(x) \leq \frac{1}{(\beta+s)^{\varrho} \Gamma(\varrho)} \\
& \quad \cdot\left[\left(x^{\beta+s}-x_{0}^{\beta+s}\right)^{\varrho} M_{\varphi}^{f}\left(x_{0}, x\right)+\left(y_{0}^{\beta+s}-x^{\beta+s}\right)^{\varrho} M_{\varphi}^{f}\left(x, y_{0}\right)\right] .
\end{aligned}
$$

Corollary 10. Using $\Psi(t)=\Gamma(\varrho) t^{\mathrm{e} / k} / k \Gamma_{k}(\varrho)$ and $g(x)=$ $x^{s+1} / s+1, s>0$ in (10) and (11), respectively, with $p=\Phi=0$, then fractional integral operators $\left({ }_{k}^{s} I_{x_{0}^{+}}^{\mathrm{Q}} f\right)(x)$ and $\left({ }_{k}^{s} I_{y_{0}^{-}}^{\mathrm{\rho}} f\right)(x)$ given in [28] are obtained which satisfy the following bound:
Corollary 12. Using $\Psi(t)=t^{\varrho}, \varrho>0, g(x)=\left(x-x_{0}\right)^{\varepsilon} / \varepsilon$ in (10), and $g(x)=-\left(y_{0}-x\right)^{\varepsilon} / \varepsilon$ in (11), where $\varepsilon>0$ with $p=\Phi=0$, then following fractional integral operators are obtained given in [12]:

$$
\begin{aligned}
& \left(F_{\lambda, \delta, \kappa, x_{0}^{+}}^{t^{\varrho}, \gamma, 9, \chi} f\right)(x)=\left({ }^{\varepsilon} I_{x_{0}^{+}}^{\varrho} f\right)(x)=\frac{\varepsilon^{1-\varrho}}{\Gamma(\varrho)} \int_{x_{0}}^{x}\left(\left(x-x_{0}\right)^{\varepsilon}-\left(t-x_{0}\right)^{\varepsilon}\right)^{\varrho-1}\left(t-x_{0}\right)^{\varepsilon-1} f(t) \mathrm{d} t \\
& \left(F_{\lambda, \delta, \kappa, y_{0}^{\prime}}^{t^{e}, \gamma, 9, \kappa, \chi} f\right)(x)=\left({ }^{\varepsilon} I_{y_{0}^{-}}^{\varrho} f\right)(x)=\frac{\varepsilon^{1-\varrho}}{\Gamma(\varrho)} \int_{x}^{y_{0}}\left(\left(y_{0}-x\right)^{\varepsilon}-\left(y_{0}-t\right)^{\varepsilon}\right)^{\varrho-1}\left(y_{0}-t\right)^{\varepsilon-1} f(t) \mathrm{d} t .
\end{aligned}
$$

Furthermore, the following bound is also satisfied:

$$
\begin{aligned}
\left({ }^{\varepsilon} I_{x_{0}^{+}}^{\varrho} f\right)(x)+\left({ }^{\varepsilon} I_{y_{0}^{-}}^{\varrho} f\right)(x) & \leq \frac{1}{\varepsilon^{\varrho} \Gamma(\varrho)} \\
\cdot & {\left[\left(x-x_{0}\right)^{\varepsilon \varrho} M_{\varphi}^{f}\left(x_{0}, x\right)+\left(y_{0}-x\right)^{\varepsilon \varrho} M_{\varphi}^{f}\left(x, y_{0}\right)\right] . }
\end{aligned}
$$

Corollary 13. For $\Psi(t)=\Gamma(\varrho) t^{\mathrm{\rho} / k} / k \Gamma_{k}(\varrho), \varrho>k$ and $g(x)=\left(x-x_{0}\right)^{\varepsilon} / \varepsilon$ in (10) and $g(x)=-\left(y_{0}-x\right)^{\varepsilon} / \varepsilon$ in (11), where $\varepsilon>0$ with $p=\Phi=0$, then the following fractional integral operators are obtained given in [29]:

$$
\begin{aligned}
\left(F_{\lambda, \delta, \kappa, x_{0}^{+}}^{\left(\Gamma(\varrho) \ell^{\rho / k} / k \Gamma_{k}(\varrho)\right), \gamma, \vartheta, \kappa, \chi} f\right)(x)=\left({ }_{k}^{\varepsilon} I_{x_{0}^{+}}^{\varrho} f\right)(x) & =\frac{\varepsilon^{1-(\varrho / k)}}{k \Gamma_{k}(\varrho)} \int_{x_{0}}^{x}\left(\left(x-x_{0}\right)^{\varepsilon}-\left(t-x_{0}\right)^{\varepsilon}\right)^{(\varrho / k)-1}\left(t-x_{0}\right)^{\varepsilon-1} f(t) \mathrm{d} t, \\
\left(F_{\lambda, \delta, \kappa, y_{0}^{-}}^{\left(\Gamma(\varrho) \rho \Gamma^{\rho / k} / k(\varrho)\right), \gamma, \vartheta, \kappa, \chi} f\right)(x) & =\left({ }_{k}^{\varepsilon} I_{y_{0}^{-}}^{\varrho} f\right)(x)=\frac{\varepsilon^{1-(\varrho / k)}}{k \Gamma_{k}(\varrho)} \int_{x}^{y_{0}}\left(\left(y_{0}-x\right)^{\varepsilon}-\left(y_{0}-t\right)^{\varepsilon}\right)^{(\varrho / k)-1}\left(y_{0}-t\right)^{\varepsilon-1} f(t) \mathrm{d} t .
\end{aligned}
$$

Furthermore, the following bound is also satisfied:

$$
\left({ }_{k}^{\varepsilon} I_{x_{0}^{+}}^{\varrho} f\right)(x)+\left({ }_{k}^{\varepsilon} I_{y_{0}^{-}}^{\varrho} f\right)(x) \leq \frac{1}{\varepsilon^{\varrho / k} k \Gamma_{k}(\varrho)}\left[\left(x-x_{0}\right)^{\varepsilon \rho / k} M_{\varphi}^{f}\left(x_{0}, x\right)+\left(y_{0}-x\right)^{\varepsilon \rho / k} M_{\varphi}^{f}\left(x, y_{0}\right)\right] .
$$


Similar bounds can be obtained for Theorems 7 and 8 which we leave for the reader.

\section{Concluding Remarks}

A notion namely $\varphi$-quasiconvexity is studied under an integral operator that associates with different kinds of operators independently defined by various authors during the last two decades. The consequences of the results are compiled in the form of corollaries and remarks. Although some of the particular cases are analyzed in Section 3 by applying Theorem 5, the reader can further compute more results as desired by applying other theorems.

\section{Data Availability}

There are no additional data required for the finding of results of this paper.

\section{Disclosure}

There is no funding available for the publication of this paper.

\section{Conflicts of Interest}

The authors declare that there are no conflicts of interest.

\section{Authors' Contributions}

All authors have equal contribution in this article.

\section{Acknowledgments}

This work was supported by the Dong-A University Research Fund.

\section{References}

[1] G. Adilov and I. Yesilce, "On generalization of the concept of convexity," Hacettepe Journal of Mathematics and Statistics, vol. 41, no. 5, pp. 723-730, 2012.

[2] G. Adilov and I. Yesilce, " $\mathrm{B}^{-1}$-Convex sets and $\mathrm{B}^{-1}$-Measurable maps," Numerical Functional Analysis and Optimization, vol. 33, no. 2, pp. 131-141, 2012.

[3] G. A. Anastassiou, "Generalized fractional Hermite-Hadamard inequalities involving $m$-convexity and $(\mathrm{s}, \mathrm{m})$-convexity," Series: Mathematics and Informatics, vol. 28, no. 2, pp. 107-126, 2013.

[4] W. Briec and C. Horvath, "B-Convexity," Optimization, vol. 53, no. 2, pp. 103-127, 2004.

[5] M. Bombardelli and S. Varošanec, "Properties of $h$-convex functions related to the Hermite-Hadamard-Fejér inequalities," Computers \& Mathematics with Applications, vol. 58, no. 9, pp. 1869-1877, 2009.

[6] H. Hudzik and L. Maligranda, "Some remarks on s-convex functions," Aequationes Mathematicae, vol. 48, no. 1, pp. 100-111, 1994.

[7] Y. C. Kwun, M. Zahra, G. Farid, S. Zainab, and S. M. Kang, "On a unified integral operator for $\varphi$-convex functions," Advances in Difference Equations, vol. 297, 2020.
[8] V. G. Mihesan, "A generalization of convexity,” in Proccedings of the Seminar on Functional Equations, Approx. And Convex, Cluj-Napoca, Romania, 1993.

[9] M. E. Özdemir, A. O. Akdemri, and E. Set, "On (h,m)convexity and Hadamard type inequalities," Journal of Mathematics and Mechanics, vol. 8, no. 1, pp. 51-58, 2016.

[10] S.-M. Yuan and Z.-M. Liu, "Some properties of $\alpha$-convex and $\alpha$-quasiconvex functions with respect to n-symmetric points," Applied Mathematics and Computation, vol. 188, no. 2, pp. 1142-1150, 2007.

[11] G. Farid, "Existence of an integral operator and its consequences in fractional and conformable integrals," Open Journal of Mathematical Sciences, vol. 3, no. 1, pp. 210-216, 2019.

[12] F. Jarad, E. Ugurlu, T. Abdeljawad, and D. Baleanu, "On a new class of fractional operators," Advances in Difference Equations, vol. 2017, p. 247, 2017.

[13] T. U. Khan and M. A. Khan, "Generalized conformable fractional operators," Journal of Computational and Applied Mathematics, vol. 346, pp. 378-389, 2019.

[14] A. A. Kilbas, H. M. Srivastava, and J. J. Trujillo, Theory And Applications Of Fractional Differential Equations, Vol. 204, Elsevier, New York, NY, USA, 2006.

[15] G. Rahman, D. Baleanu, M. A. Qurashi, S. D. Purohit, S. Mubeen, and M. Arshad, "The extended Mittag-Leffeler function via fractional calculus," Journal of Nonlinear Science and Applications, vol. 10, pp. 4244-4253, 2013.

[16] S. S. Dragomir and C. E. M. Pearce, "Quasi-convex functions and Hadamard's inequality," Bulletin of the Australian Mathematical Society, vol. 57, no. 3, pp. 377-385, 1998.

[17] G. Farid, C. Y. Jung, S. Ullah, W. Nazeer, M. Waseem, and S. M. Kang, "Some generalized $k$-fractional integral inequalities for quasi-convex functions," Journal of Computational Analysis and Applications, vol. 29, no. 3, pp. 454-467, 2021.

[18] S. Ullah, G. Farid, K. A. Khan, A. Waheed, and S. Mehmood, "Generalized fractional inequalities for quasi-convex functions," Advance Difference Equations, vol. 15, 2019.

[19] D. Zhao, G. Farid, M. Zeb, S. Ahmad, and K. Mahreen, "On boundedness of unified integral operators for quasiconvex functions," Advance Difference Equations, vol. 38, 2020.

[20] A. W. Roberts and D. E. Varberg, Convex Functions, Academic Press, New York, NY, USA, 1973.

[21] M. E. Gordji, M. R. Delavar, and M. D. L. Sen, "On $\varphi$-convex functions," Journal of Mathematical Inequalities, vol. 10, no. 1, pp. 173-183, 2016.

[22] D. A. Ion, "Some estimates on the Hermite-Hadamard inequality through quasi-convex functions," Analele Universitatii din Craiova. Seria Matematica, vol. 34, pp. 82-87, 2007.

[23] Y. C. Kwun, G. Farid, W. Nazeer, S. Ullah, and S. M. Kang, "Generalized Riemann-Liouville $k$-fractional integrals associated with Ostrowski type inequalities and error bounds of Hadamard inequalities," IEEE Access, vol. 6, 2018.

[24] M. Andrić, G. Farid, and J. Pečarić, "A further extension of Mittag-Leffler function," Fractional Calculus and Applied Analysis, vol. 21, no. 5, pp. 1377-1395, 2018.

[25] Y. C. Kwun, G. Farid, W. Nazeer, S. Ullah, K. Mahreen, and S. M. Kang, "Inequalities for a unified integral operator and associated results in fractional integrals," IEEE Access, vol. 7, 2019.

[26] S. Mubeen and G. M. Habibullah, " $\kappa$-fractional integral and application," International Journal of Contemporary Mathematical Sciences, vol. 7, pp. 89-94, 2012. 
[27] H. Chen and U. N. Katugampola, "Hermite-Hadamard and Hermite-Hadamard-Fejér type inequalities for generalized fractional integrals," Journal of Mathematical Analysis and Applications, vol. 446, no. 2, pp. 1274-1291, 2017.

[28] M. Z. Sarikaya, Z. Dahmani, M. E. Kiris, and F. Ahmad, "( $k$, s)-Riemann-Liouville fractional integral and applications," Hacettepe Journal of Mathematics and Statistics, vol. 1, no. 45, pp. 77-89, 2016.

[29] S. Habib, S. Mubeen, and M. N. Naeem, "Chebyshev type integral inequalities for generalized $k$-fractional conformable integrals," Journal of Inequalities and Special Functions, vol. 9, no. 4, pp. 53-65, 2018. 